\title{
Differenzialdiagnose Rückenschmerzen mit Entzündungsparametern: Fallvorstellung: Manifestation einer CPPD als Crowned Dens Syndrom mit Befall peripherer Gelenke Differential Diagnosis of Back Pain with Inflammatory Markers: Case Presentation: Manifestation of CPPD as Crowned Dens Syndrome with Involvement of Peripheral Joints
}

Autoren

Arnd Kleyer $^{1}$, Frank Roemer ${ }^{2}$, Georg Schett ${ }^{1}$, Bernhard Manger ${ }^{1}$

Institute

1 Rheumatologie, Universitätsklinikum Erlangen, Medizinische Klinik 3, Rheumatologie und Immunologie, Erlangen, Deutschland

2 Radiologie, Universitätsklinikum Erlangen, Radiologisches Institut, Erlangen, Deutschland

Schlüsselwörter

Chondrokalzinose, CPPD, Kristallarthritis, Crowned dens syndrom, Pseudogicht

Key words

chondrocalcinosis, CPPD, crystal arthropathies, crowned dens syndrome, pseudogout

Bibliografie

Akt Rheumatol 2021; 46: 76-79

DOI https://doi.org/10.1055/a-1327-1424

ISSN 0341-051X

(C) 2021. Thieme. All rights reserved.

Georg Thieme Verlag KG, Rüdigerstraße 14,

70469 Stuttgart, Germany

Korrespondenzadresse

Dr. Arnd Kleyer

Universitätsklinikum Erlangen

Medizinische Klinik 3

Rheumatologie und Immunologie

Ulmenweg 18

91052 Erlangen

Deutschland

Tel.: +49-(0)9131-85 32093, Fax: +49-(0)9131-85 32092

arnd.kleyer@uk-erlangen.de

\section{ZUSAMMENFASSUNG}

Der aktuelle Fall beschreibt den Fall eines 84 jährigen Patienten mit zunächst unklaren Rückenschmerzen und hohen Entzündungsparameter. Nach Ausschluss infektiöser Ursache konnte im Verlauf eine CPPD (Calcium pyrophosphat deposition disease) Erkrankung mit Wirbelsäulenbefall diagnostiziert werden. Neue radiologische Segmentierungsverfahren erlaubten zudem die Detektion von CPPD Kristallen in peripheren Gelenken. Nach Gabe von Prednisolon und Kineret konnte die Beschwerdesymptomatik erfolgreich behandelt werden.

\section{ABSTRACT}

This case report describes the case of a patient with back pain of unknown origin and high inflammatory markers. After an infectious cause was ruled out, he was diagnosed with CPPD disease with spinal infestation. New radiological segmentation methods also allowed the detection of CPPD crystals in peripheral joints. Treatment with prednisolone and short-term Kineret was initiated and the symptoms were treated successfully. 


\section{Anamnese}

Die stationäre Aufnahme des 84 jährigen Patienten erfolgte zum Ausschluss einer Spondylodiszitis bei starken, teilweise immobilisierenden Schmerzen in der Halswirbelsäule sowie erhöhten Entzündungsparametern. An relevanten Vorerkrankungen bestanden eine arterielle Hypertonie, benigne Prostatahyperplasie sowie eine Angststörung. Begleitmedikation mit Tamsulosin, Amytryptilin, Sertralin, Pregabalin.

2 Wochen vor stationärer Einweisung hatte unser Patient unter akut aufgetretenen Schulterschmerzen gelitten, welche mittels symptomatischer Maßnahmen und nicht steroidalen Antiphlogistika unter ambulanten Bedingungen zunächst kontrollierbar waren. Nachdem jedoch stärkste Schmerzen in der Halswirbelsäule hinzugekommen waren, erfolgte die stationäre Aufnahme zur weiteren Differenzialdiagnostik und zum Ausschluss einer Spondylodiszitis.

\section{Diagnostik}

\section{Klinischer Befund}

Reduzierter Allgemeinzustand, guter Ernährungszustand; Größe $182 \mathrm{~cm}$, Gewicht $90 \mathrm{~kg}$, Cor/Pulmo/Abdomen: unauffälliger Untersuchungsbefund, diskrete Beinödeme beidseits. Rechte Schulter/ rechtes Schulterblatt: muskulärer Hartspann, Schmerzen im Ruhe und bei Bewegung; dumpfer Schmerzcharakter, keine Einschränkung der Kraft und Beweglichkeit. Halswirbelsäule: Steifigkeit insbesondere Morgens (30 min), nächtliche Schmerzen und Myalgien im HWS/Nakenbereich.

\section{Initiale radiologische Diagnostik}

Die Spondylodiszitis konnte durch eine initial durchgeführte Magnetresonanztomografie der Wirbelsäule ausgeschlossen werden.
Es zeigte sich jedoch ein Ödem mit Begleitreaktion um HWK 7 und BWK 1 mit umgebender entzündlicher Begleitreaktion der Weichteilstrukturen bei bereits vorbekannten foraminalen Engen von HWK 3 bis BWK 1.

Labordiagnostik: Serologisch fanden sich keine Hinweise auf eine rheumatologische Ursache der Gelenkbeschwerden. (RF/Anti-CCP/ ANAs/ANCAs) zeigten sich unauffällig. Harnsäure $(3,8 \mathrm{mg} / \mathrm{dl})$ Nierenretentionsparameter (Kreatinin $0,81 \mathrm{mg} / \mathrm{dl}$ ), Elektrolyte (Kalium/Calcium/Natrium/Magnesium) waren ebenfalls im Normbereich.

Weiterführende radiologische Diagnostik: Aufgrund der vorbeschriebenen ausgeprägten degenerativen Veränderungen der HWS führten wir eine CT Untersuchung durch, bei der sich eine Verkalkung der Ligamenta alaria um den Dens axis ( $>$ A b b. 1) sowie der Supraspinatussehe beidseits zeigte ( $\bullet$ Abb. 2 ), sodass wir den Verdacht auf eine Kalziumpyrophosphaterkrankung mit einem akuten Schub stellten.

Zum Abklärung weitere Gelenkmanifestationen wurden Röntgenbilder der Kniegelenke sowie der Schultern durchgeführt. Am linken Kniegelenk zeigte sich eine Meniskocalzinose in lateralen Kompartiment. In der Schulter zeigten sich Verkalkungen im Bereich der Supraspinatussehne.

Neben den großen Gelenken kann es häufig zu einem Befall der mittleren und kleineren Gelenke kommen. Insbesondere der radiocarpale und radio-ulnare Bereich mit dem TFCC ist häufig befallen [1-5]. Um dies bei unserem Patienten auszuschließen wurde eine konventionelle Röntgenaufnahme der Hand durchgeführt ( $\triangleright$ Abb. 3). Hier zeigte sich kein eindeutiger Hinweis auf Kalkablagerungen. Bei persistierenden Schmerzen führten wir eine hochauflösende CT Aufnahme durch. Erst hiermit konnte insbesondere unter Zuhilfenahme neuer fotorealistischen Segmentierungstechniken (Cinematic Rendering, Siemens Healthineers) der Befall der Handgelenke mit CPPD Kristallen sichtbar gemacht werden ( Abb. 3) [6].
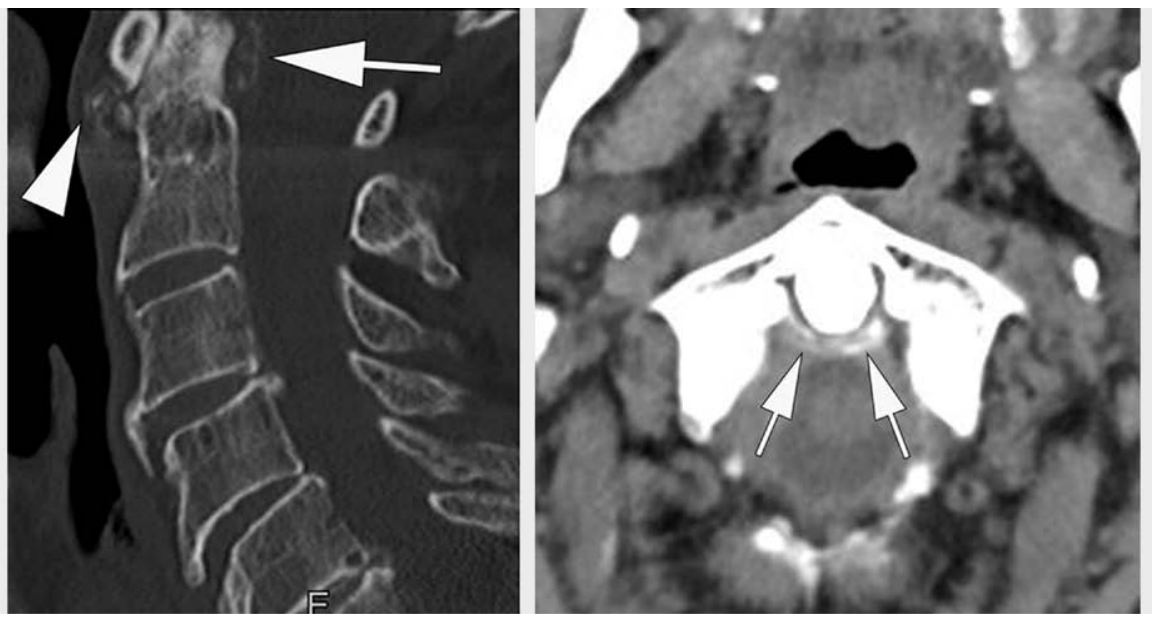

- Abb. 1 Konventionelle CT Aufnahme der Halswirbelsäule. Sagittale (links) und coronare (rechts) Reformationen. Weiße Pfeile deuten auf Bandförmige Verkalkungen um die Densspitze entlang des periodontalen Ligaments mit sog. „crown-sign. Die Pfeilspitze zeigt zusätzliche Verkalkungen der anterioren Bandstrukturen. 

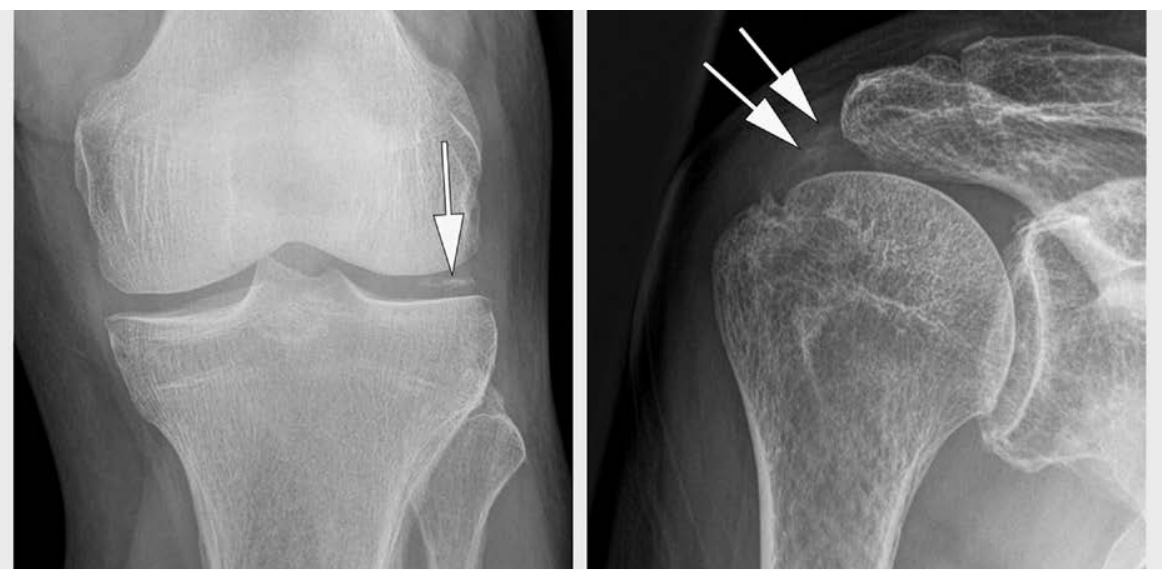

- Abb. 2 Konventionelle Röntgenaufnahmen. Im Bereich des lateralen Gelenkspaltes des linken Kniegelenks sowie im Bereich der Rotatorenmanschette der rechten Schulter (weiße Pfeile) deutliche Zeichen einer Verkalkung
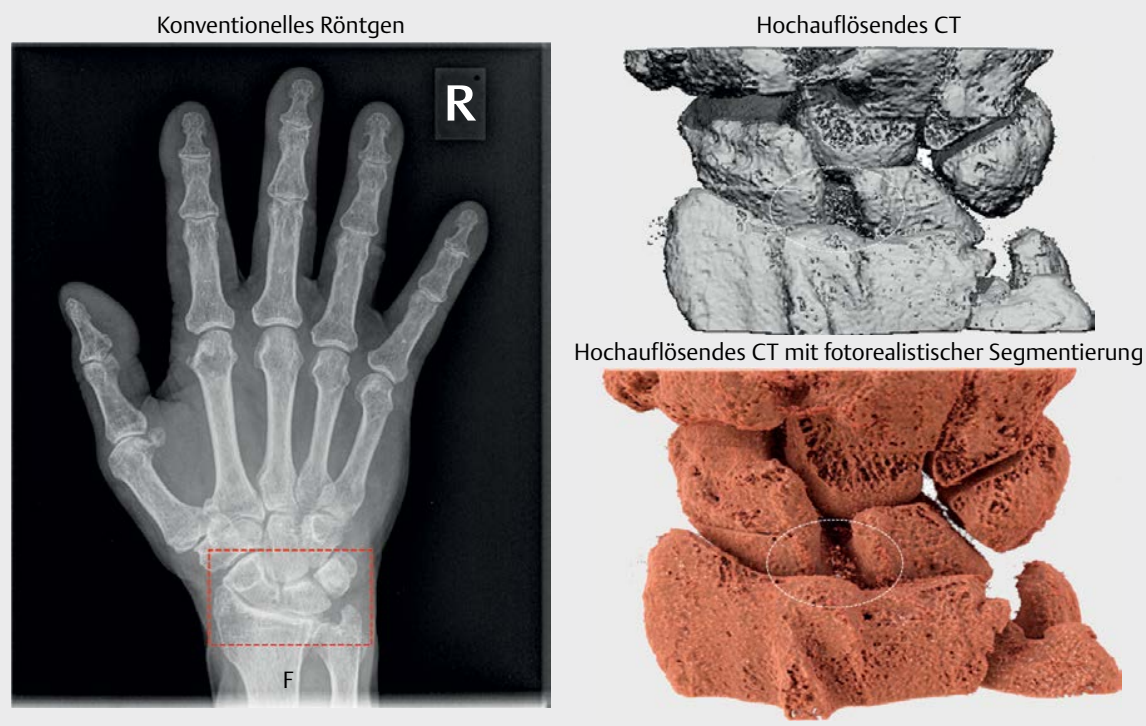

Abb. 3 Konventionelle Röntgenaufnahme: Im Bereich der proximalen Handgelenkesreihe ist im konventionellen Röntgen kein Hinweis auf Kalkablagerungen zu sehen. Auf der rechten Seite sind segmentierte Aufnahmen aus einen HR-pQCT Scanner zu sehen.In der oberen Reihe eine konventionelle Segmentierung, in der unteren Reihe eine neue, fotorealistische Segmentierung des Handgelenks. Hier finden sich innerhalb der weißen Kreise deutliche Nachweise von Kalkablagerungen.

\section{Diskussion}

\section{Herausforderung in der Diagnostik}

Die CPPD kann neben den peripheren Gelenken auch das Achsenskelett befallen. Das „crowned dens syndrom“ stellt wohl die bekannteste Sonderform der axialen CPPD nämlich die Beteiligung des Atlantodentalgelenks „crowned dens sign“ dar [7-9]. Die diagnostische Herausforderung besteht in der richtigen Einschätzung der häufig geäußerten Symptome mit Nackenschmerzen (in 100\% der Fälle) und -steifigkeit (in 98\%) in Verbindung mit Fieber (in $80 \%$ ) und hohen Entzündungsparametern (in 88\%) [7]. Differenzialdiagnostisch wird zunächst an Erkrankungen wie Meningitis, Polymyalgia rheumatica, Riesenzellarteriitis oder Spondylodiszitis gedacht [9]. Für die Diagnostik der CPPD an der Wirbelsäule ist die
Computertomografie das bildgebende Verfahrung der Wahl, da nur mit dieser Bildgebung die linearen Kalzifizierungen um den Dens axis - meist im Ligamentum transversum gelegen -nachgewiesen werden können [10].

\section{Therapeutische Maßnahmen}

Leider stellt das Fehlen aussagekräftiger, kontrollierter Studien den behandelnden Arzt vor eine große Herausforderung. In Anlehnung an die Behandlung eines Schubes einer Arthritis Urica werden viele Therapieansätze in Analogie verwendet zur Behandlung einer symptomatischen CPPD. Die europäischen Fachgesellschaft EULAR der Rheumatologen veröffentlichte 2011 Empfehlungen zum Management dieser Erkrankung, basierend auf der (geringen) verfügbaren Evidenz [11]. 
So umfasst die Behandlung medikamentöse und nichtmedikamentöse Maßnahmen und sollte auch Komorbiditäten und metabolische Grunderkrankungen miteinschließen. Im Falle eines Schubes einer CPPD Arthritis können Ruhe, Kühlung und Gelenkinjektion mit Glukokortikoiden ausreichend sein. SowohI NSAR als auch Colchicin (3-4 msl 0,5 mg/dl) sind wirksam in der Therapie der akuten CPPD Arthritis. Darüber hinaus ist eine kurze Therapie mit oralen Kortikosteroiden in absteigender Dosierung möglich. Als Anfallsprophylaxe bei häufig rezidivierenden akuten CPP-Arthritiden kommt Colchicin $(0,5-1 \mathrm{mg} / \mathrm{d})$ oder niedrig dosierten NSAR möglich. In Fallberichten ist zudem der kurzfristige Einsatz von IL1 Blockern wir Kineret bei therapierefraktärer Erkrankung möglich [11-13].

\section{Therapie und Verlauf im aktuellen Fall}

Nach Ausschluss der Spondylodiszitis erfolgte die Gabe von Methylprednisolon $100 \mathrm{mg}$ i.v (SDH) mit anschließender Reduktion auf $20 \mathrm{mg}$ Prednisolon p.o. und NSAR (Etoricoxib $60 \mathrm{mg} / \mathrm{ag}$ ). Darüber hinaus erfolgte die Gabe von Kineret (Anakinra) über 3 Tage mit einer Gabe s.c./Tag. Hierunter sistierten die Beschwerden prompt. Bis zur Entlassung konnte die Prednisolondosis auf 7,5 mg/Tag reduziert werden mit der Empfehlung das Prednisolon innerhalb von 4 Wochen schrittweise abzusetzen. Bei Entlassung war der Patient beschwerdefrei.

\section{Funding}

This study was supported by a project grant from Rheumastiftung (Neue Konzepte zur Diagnostik und Therapie der Chondrokalzinose), Siemens Healhineers provided cinematic rendering terchnique.

\section{Danksagung}

Klaus Engel (Healthineers) Unterstützung Cinematic Rendering.

\section{Interessenkonflikt}

Die Autoren (AK) erklären, dass sie innerhalb der vergangenen 3 Jahre Forschungsunterstützung von Lilly Pharma, Novartis und Gilead sowie Vortragshonorare von Novartis, Lilly, Gilead, Janssen erhalten haben.
[1] Zhang W, Doherty M, Bardin T et al. European League Against Rheumatism recommendations for calcium pyrophosphate deposition. Part I: terminology and diagnosis. Ann Rheum Dis 2011; 70: 563-570

[2] Rosenthal AK, Ryan LM. Calcium Pyrophosphate Deposition Disease. N Engl J Med 2016; 374: 2575-2584

[3] Cho NH, Song $Y$, Lee $S$ et al. Incidence of knee chondrocalcinosis and its risk factors in a community-based cohort. Int J Rheum Dis 2018; 21: 1391-1397

[4] Filippou G, Scirè CA, Adinolfi A et al. Identification of calcium pyrophosphate deposition disease (CPPD) by ultrasound: reliability of the OMERACT definitions in an extended set of joints-an international multiobserver study by the OMERACT Calcium Pyrophosphate Deposition Disease Ultrasound Subtask Force. Ann Rheum Dis 2018; 77: 1194-1199

[5] Filippou G, Scirè CA, Damjanov N et al. Definition and Reliability Assessment of Elementary Ultrasonographic Findings in Calcium Pyrophosphate Deposition Disease: A Study by the OMERACT Calcium Pyrophosphate Deposition Disease Ultrasound Subtask Force. J Rheumatol 2017; 44: 1744-1749

[6] Glemser PA, Engel K, Simons D et al. A New Approach for Photorealistic Visualization of Rendered Computed Tomography Images. World Neurosurg 2018; 114: e283-e292

[7] Oka A, Okazaki K, Takeno A et al. Crowned Dens Syndrome: Report of Three Cases and a Review of the Literature. J Emerg Med 2015; 49: e9-e13

[8] Koda R, Tsuchida Y, Yoshizawa K et al. Crowned Dens Syndrome as an Initial Manifestation of Crystalline Deposition Disease. Intern Med 2015; 54: 2405-2408

[9] Bridges KJ, Bullis CL, Wanchu A et al. Pseudogout of the cervical and thoracic spine mimicking infection after lumbar fusion: case report. J Neurosurg Spine 2017; 27: 145-149

[10] Moses V, Parmar HA, Sawalha AH. Magnetic Resonance Imaging and Computed Tomography in the Evaluation of Crowned Dens Syndrome Secondary to Calcium Pyrophosphate Dihydrate. J Clin Rheumatol 2015; 21: 368-369

[11] Zhang W, Doherty M, Pascual E et al. EULAR recommendations for calcium pyrophosphate deposition. Part II: management. Ann Rheum Dis 2011; 70: 571-575

[12] Andrés M, Sivera F, Pascual E. Therapy for CPPD: Options and Evidence. Curr Rheumatol Rep 2018; 20: 31

[13] Cipolletta E, Di Matteo A, Scanu A, et al., Biologics in the treatment of calcium pyrophosphate deposition disease: a systematic literature review. Clin Exp Rheumatol 2020 